\title{
Giant vortices, rings of vortices, and reentrant behavior in type-1.5 superconductors
}

\author{
V. H. Dao, ${ }^{1,2}$ L. F. Chibotaru, ${ }^{1}$ T. Nishio,,${ }^{1,3}$ and V. V. Moshchalkov ${ }^{1, *}$ \\ ${ }^{1}$ Institute for Nanoscale Physics and Chemistry (INPAC), Katholieke Universiteit Leuven, Celestijnenlaan 200F, B-3001 Leuven, Belgium \\ ${ }^{2}$ Centre de Physique Moléculaire Optique et Hertzienne, UMR 5798 du CNRS, Université Bordeaux 1, \\ 351 cours de la Libération, F-33405 Talence, France \\ ${ }^{3}$ Department of Physics, Tokyo University of Science, Kagurazaka, Shinjuku, Tokyo 162-8601, Japan
}

(Received 9 September 2010; published 24 January 2011)

\begin{abstract}
We predict that in a bulk type-1.5 superconductor, the competing magnetic responses of the two components of the order parameter can result in group-stabilized giant vortices and individual rings of vortices in the absence of any extrinsic pinning or confinement mechanism. We also determine within the Ginzburg-Landau theory a condition for the robustness of type 1.5 in the vicinity of the critical temperature, and we find a rich phase diagram with successions of behaviors such as type $1 \rightarrow$ type $1.5 \rightarrow$ type $2 \rightarrow$ type 1.5 when temperature decreases.
\end{abstract}

DOI: 10.1103/PhysRevB.83.020503 PACS number(s): 74.25.Ha, 11.27.+d, 74.25.Uv, 74.70.Ad

Topological defects have been intensively investigated in a wide variety of physical systems, for example, in condensed matter (ferromagnets, superfluids, liquid crystals, etc.), ${ }^{1}$ optics, ${ }^{2}$ particle physics, ${ }^{3}$ and astrophysics. ${ }^{4}$ In a superconductor, the quantized line defect is a vortex (also called flux line), which carries a localized integer multiple of the magnetic flux quantum $\Phi_{0}=h / 2 e$. Close to the transition temperature, a unique quantity, the Ginzburg-Landau (GL) parameter $\kappa$, determines superconductivity response to a magnetic field. ${ }^{5}$ It is equal to the ratio of the magnetic penetration depth $\lambda$ to the coherence length $\xi$ of the order parameter (OP) and can be calculated from the material parameters within the microscopic BCS theory. In a type-1 superconductor, $\kappa<1 / \sqrt{2}$, the interaction between vortices is purely attractive, which results in their fusion into macroscopic normal domains in the intermediate state. In contrast, in a type-2 superconductor, $\kappa>1 / \sqrt{2}$, the interaction is purely repulsive, so the vortices form a stable lattice of few lines in the mixed state. At the critical value, the GL theory (which is mathematically equivalent to the Abelian Higgs model in particle physics) predicts that the vortex interaction is exactly zero. 6,7 However, experiments with $\kappa \approx 1 / \sqrt{2}$ showed complex patterns consisting of mixed-state domains with vortex-free Meissner domains. ${ }^{5,8}$ This stems from a nonmonotonic intervortex potential that is repulsive at short separation and attractive at a long range. Such interaction profile can be explained theoretically by taking into account different mechanisms such as low-temperature corrections ${ }^{5,9}$ or fluctuations and anisotropy in the vortex lattice. ${ }^{10}$

Recently, Babaev and Speight ${ }^{11}$ predicted that in a superconductor with two uncoupled components the intervortex potential can be nonmonotonic when one component of the OP is of type 1 while the other is of type 2 . This idea has been recently supported by observations ${ }^{12}$ of highly inhomogeneous vortex distributions in clean samples of two-band superconductor $\mathrm{MgB}_{2} \cdot{ }^{13}$ The estimates of $\mathrm{MgB}_{2}$ parameters ${ }^{12}$ indicate that this behavior, coined type- 1.5 superconductivity, only occurs in high-quality samples, which explains why the material was classified before as type 2 . Including the finite interband Josephson coupling (which is moderate in $\mathrm{MgB}_{2}$ ), GL calculations yielded nonmonotonic vortex interaction potentials, also when one of the bands is inactive ${ }^{12}$ (in this case, although the OP components vary on the same characteristic length scale at a long range, their variations are dissimilar at a finite distance from the vortex core ${ }^{14}$ ). Stimulated by the growing number of discovered multiband superconductors, for example, iron-based superconductors, ${ }^{15,16}$ we investigate in this article features of the type- 1.5 regime that distinguish it from single-component superconductivity at the critical $\kappa$. We find, in particular, group-stabilized giant vortices and vortex rings composed of single- and multiquantum vortices as thermodynamically stable phases.

The two-band GL theory. In the context of two-band superconductivity with s-wave symmetry of the OP, we consider the GL free-energy functional ${ }^{17}$

$$
\mathcal{F}_{\mathrm{GL}}=\mathcal{F}_{1}+\mathcal{F}_{2}-\gamma\left(\Psi_{1}^{*} \Psi_{2}+\Psi_{1} \Psi_{2}^{*}\right)+\frac{1}{8 \pi}(\nabla \times \mathbf{A})^{2},
$$

where the contributions from each band

$\mathcal{F}_{n}=\alpha_{n}\left|\Psi_{n}\right|^{2}+\frac{\beta_{n}}{2}\left|\Psi_{n}\right|^{4}+\frac{1}{2 m_{n}}\left|\left(-i \nabla+\frac{2 \pi}{\Phi_{0}} \mathbf{A}\right) \Psi_{n}\right|^{2}$

are supplemented by a Josephson-type coupling and the magnetic energy. The Josephson interaction describes Cooper pair tunneling between the two bands. For example, in $\mathrm{MgB}_{2}$ the other terms mixing the two components $\Psi_{n}$ of the OP vanish in the clean limit. ${ }^{17,18}$ We do not investigate the dirty limit that we expect to be detrimental to type-1.5 superconductivity [scattering by impurities tends (i) to average out the multiband character and (ii) to turn a type- 1 into a type- 2 superconductor], so we omit the latter terms here. In contrast to other kinds of superfluid mixture, for example, proton and neutron Cooper pairs in neutron stars, ${ }^{4}$ the Josephson term is zero while the Andreev-Bashkin cross-gradient term is important. ${ }^{19}$ Although $\alpha_{1}$ and $\alpha_{2}$ can change sign at different temperatures, any finite Josephson coupling $\gamma$ forces both $\Psi_{n}$ to vanish at the same critical temperature $T_{c}$. Below $T_{c}$ in the homogeneous Meissner state, $\mathbf{A}=0$ and $\left|\Psi_{n}\right|=u_{n}$, which are solutions of $u_{n}\left(\alpha_{n}+\beta_{n} u_{n}^{2}\right)-\gamma u_{3-n}=0$. The energy density is then

$$
\epsilon_{M}=-\left(\beta_{1} u_{1}^{4}+\beta_{2} u_{2}^{4}\right) / 2 .
$$

Superconductivity with two OP components is richer than with a single one because not only does the ratio $u_{2} / u_{1}$ change with temperature but also the two components can have 
different magnetic responses and spatial variations. Let us introduce the band parameters $\kappa_{n}$ and their average $\bar{\kappa}$ defined by

$$
\begin{aligned}
\kappa_{n} & =\Phi_{0} m_{n} \sqrt{\beta_{n}} /(2 \pi)^{3 / 2}, \\
\bar{\kappa}^{-1} & =\sqrt{p_{1}} \kappa_{1}^{-1}+\sqrt{p_{2}} \kappa_{2}^{-1},
\end{aligned}
$$

where the condensation-energy fraction

$$
p_{n}=\beta_{n} u_{n}^{4} / 2\left|\epsilon_{M}\right| \text {. }
$$

If the two components were separated not in band space but in real space as in a Josephson junction, $\kappa_{1}$ and $\kappa_{2}$ would be the actual GL parameters of the two superconductors in contact. Note that $\bar{\kappa}$ varies with temperature contrary to $\kappa_{n}$. To discuss the different types of vortex interaction, it is convenient to rescale the distance $x=\lambda \sqrt{2} x^{\prime}$, the vector potential $\mathbf{A}=\left(\Phi_{0} / \sqrt{2} \pi \lambda\right) \mathbf{a}$, and $\Psi_{n}=u_{n} \psi_{n}$. Here the magnetic penetration depth is defined by

$$
\lambda^{2}=\Phi_{0} \bar{\kappa} /(4 \pi)^{3 / 2}\left|\epsilon_{M}\right|^{1 / 2} .
$$

The normalized functional $\mathcal{F} \equiv\left(\mathcal{F}_{\mathrm{GL}}-\epsilon_{M}\right) / \epsilon_{\lambda}$ is then

$$
\begin{aligned}
\mathcal{F}= & \frac{1}{2 \pi}\left\{\bar{\kappa}^{2}\left[p_{1}\left(\left|\psi_{1}\right|^{2}-1\right)^{2}+p_{2}\left(\left|\psi_{2}\right|^{2}-1\right)^{2}+q\left|\psi_{1}-\psi_{2}\right|^{2}\right]\right. \\
& \left.+r_{1}\left|D \psi_{1}\right|^{2}+r_{2}\left|D \psi_{2}\right|^{2}+\frac{1}{2}(\nabla \times \mathbf{a})^{2}\right\},
\end{aligned}
$$

where the line energy $\epsilon_{\lambda}=\left(\Phi_{0} / 4 \pi \lambda\right)^{2}, D=(-i \nabla+\mathbf{a})$, the normalized Josephson coupling factor

$$
q=\gamma u_{1} u_{2} /\left|\epsilon_{M}\right|
$$

and the superfluid-density fraction

$$
r_{n}=u_{n}^{2} m_{n}^{-1} /\left(u_{1}^{2} m_{1}^{-1}+u_{2}^{2} m_{2}^{-1}\right) .
$$

So in contrast to single-component superconductivity where the shape of the vortex interaction depends only on $\kappa,{ }^{7}$ the shape depends here on four parameters: $\left\{\bar{\kappa}, p_{1}, q, r_{1}\right\}$ (since $p_{1}+p_{2}=1$ and $\left.r_{1}+r_{2}=1\right)$ or equivalently $\left\{\bar{\kappa}, \kappa_{1}, \kappa_{2}, q\right\}$. However, the Josephson term locks $\psi_{1}$ with $\psi_{2}$ when $q$ diverges to infinity at the critical temperature (since $u_{n}$ vanishes) and, as illustrated below, the single-component behavior with $\psi_{1}=\psi_{2}$ and $\kappa=\bar{\kappa}$ is recovered in this limit if $\bar{\kappa} \neq 1 / \sqrt{2}$.

Numerical evaluation of the vortex interaction. The vortexpair potential is numerically calculated by variational minimization of the GL energy $F=\int \mathcal{F} d^{2} x$ with the constraint that the pair separation $d$ is held fixed. ${ }^{20}$ The ansatz $^{7}$ for $F$ is built upon isolated-vortex solutions with winding number $L=1$ and 2. For the latter, the radial distributions $\psi_{n}(r)$ and $\mathbf{a}(r)$ are found by solving the finite-difference version of the GL equations on a linear grid of 3000 points with a relaxation method. The boundary conditions are $\left|\psi_{1}\right|=\left|\psi_{2}\right|=a_{\theta}=0$ at $r=0$ and $\left|\psi_{1}\right|=\left|\psi_{2}\right|=1, a_{\theta}=-L / \sqrt{2} r$ at large distance $r \sim 100 \lambda$.

Figure 1 shows the interaction potential $U^{v v}(d) \equiv F(d)-$ $F(\infty)$ of two vortices when one OP component is of type $1\left(\kappa_{1}=0.5\right)$ and the other one is of type $2\left(\kappa_{2}=5\right)$ while the average $\bar{\kappa}$ is $0.6,1 / \sqrt{2}$, and 1 . For $\bar{\kappa}=0.6(\bar{\kappa}=1)$, the energy of a double-quantum vortex is smaller (larger) than the energy of two isolated vortices like in a single-component type-1 (-2) superconductor. We note that this is not always the case for other choices of parameters. Besides for small $q$,

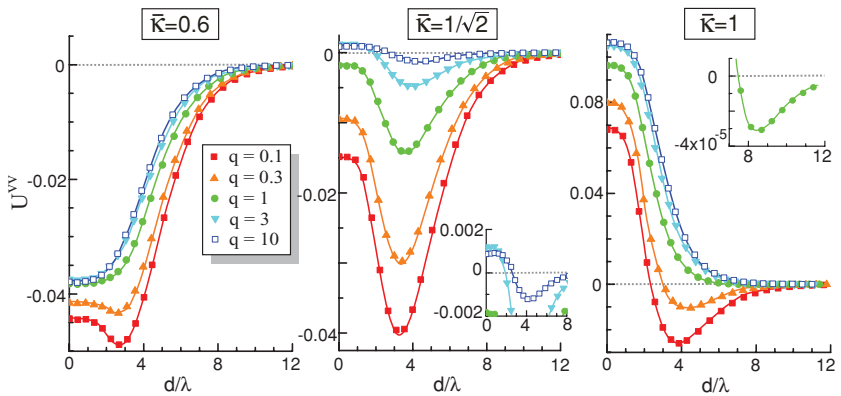

FIG. 1. (Color) Potential of the vortex interaction $U^{v v}(d) \equiv$ $F(d)-F(\infty)$ as a function of vortex separation $d$ for different values of average GL parameter $\bar{\kappa}$ (band parameters $\kappa_{1}=0.5, \kappa_{2}=5$ ) and normalized Josephson coupling $q$.

the intervortex potential is repulsive at short separation and attractive at long range even though $\bar{\kappa}$ is not in the vicinity of $1 / \sqrt{2}$. As $q$ increases, the potential is first of type 1.5 and then it recovers a type-1 $(-2)$ shape while the position $d_{\min }$ of its minimum goes to $0(+\infty)$. In contrast, the potential for $\bar{\kappa}=1 / \sqrt{2}$ is always of type 1.5 whatever the strength of the coupling.

Distribution of vortices. To illustrate the variety of magnetic responses in a type-1.5 superconductor, Fig. 2 shows the vortex patterns resulting from different interaction potentials $U^{v v}$ ranging from those more like type 1 (i) to those
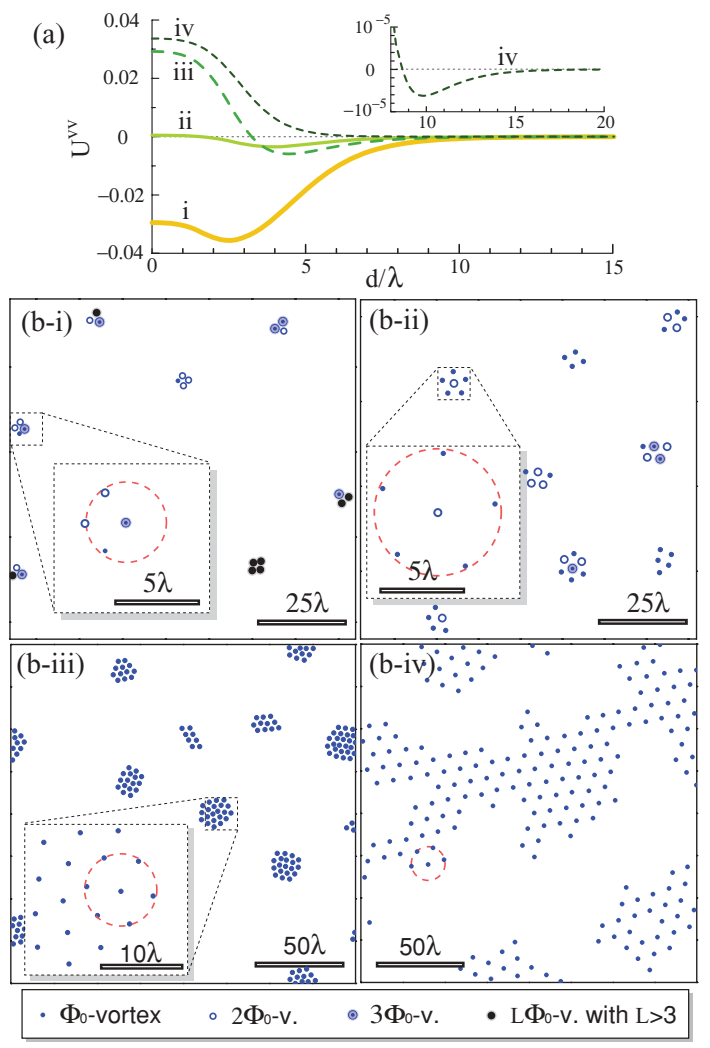

FIG. 2. (Color) (a) Different potentials of vortex interaction in a type-1.5 superconductor. The inset is a magnification of curve $i v$. (b) The vortex distributions resulting from the interaction potentials i to iv. $L \Phi_{0}$ vortex means a vortex with $L$ quanta of flux. The dashed circle represents the positions of $U^{v v}$ minimum around one vortex. 
more like type 2 (iv). They are obtained by molecular dynamics simulations of a system of overdamped vortices (see Ref. 12 for details). Starting from several random distributions, the dynamics of 200 vortices was simulated in a square of size $200 \lambda \times 200 \lambda$ with periodic boundary conditions during 100000 time steps. $^{21}$

The highly inhomogeneous vortex distributions are characteristically composed of areas filled with vortices surrounded by or surrounding vortex-free regions, depending on the ratio of the minimal-potential separation $d_{\min }$ to the average distance $d_{v v}$ between vortices [compare, e.g., (b-iii) with (b-iv) in Fig. 2]. When $d_{\min }>d_{v v}$, the whole surface is covered by a vortex lattice even though the interaction is of type 1.5. With potentials $\mathrm{i}$ and ii, a multiquantum vortex can be stabilized by the surrounding vortices at distances $\sim d_{\min }$ : the latter generate a local potential well that overcomes the repulsion between the merged vortices forming the giant vortex. However, the maximum of $U^{v v}$ at zero separation prevents all the vortices from collapsing into one unique macroscopic vortex, that is, a normal region like in the intermediate state. Giant vortices are observed in a superconductor only when stabilized by an extrinsic confinement such as boundaries in mesoscopic samples $^{22}$ or pinning sites. ${ }^{23}$ Their stability in the bulk is then a remarkable property of the type-1.5 regime. We found another feature: rings of vortices can appear, as shown in Fig. 3, when the OP components vary on length scales that are greatly different (i.e., when $\kappa_{1} \ll \kappa_{2}$ and $q \ll 1$ ). In this situation, the local maximum of the intervortex potential at zero separation is sharper than the minimum (see curves $i$ and $\mathrm{v}$ in Figs. 2 and 3), which is detrimental to the stability of a giant vortex. The favored configuration of a vortex cluster is then a annular distribution of radius $<d_{\min }$, with the presences of one central vortex and of multiquantum vortices if the bunch is numerous. Although the stabilizing mechanisms are different, these structures are reminiscent of another multicomponent superfluid, ${ }^{3} \mathrm{He}-\mathrm{A}$, where another kind of double-quantum vortex is observed in the $\mathrm{A}$ phase $\mathrm{e}^{24}$ and rings of vortices are predicted near the transition between the A and B phases. ${ }^{25}$

Limit of large normalized Josephson coupling. The robustness of type- 1.5 superconductivity at $\bar{\kappa} \approx 1 / \sqrt{2}$ toward the large coupling can be analytically demonstrated. When $q$ is large, the difference $\left(\psi_{1}-\psi_{2}\right)$ is small so the GL functional (8) can be minimized with a perturbative expansion in powers of $q^{-1}$ by writing $\psi_{1,2}=\varphi_{0} \pm \varphi_{1} / 2 q$. At zeroth order in $q^{-1}$, $\varphi_{0}$ and $a$ are then solutions of the Bogomolnyi-Jacobs-Rebbi
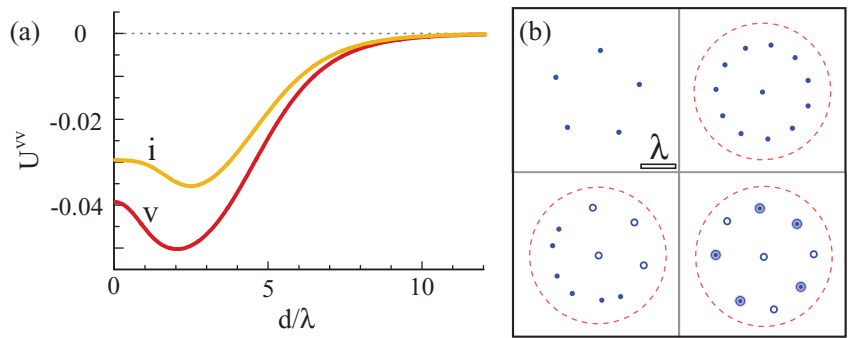

FIG. 3. (Color) (a) Vortex-interaction potentials for $\left\{\bar{\kappa}, \kappa_{1}\right.$, $\left.\kappa_{2}, q\right\}=\{0.65,0.5,25,0.4\} \quad$ (curve i) and $\{0.83,0.5,100,0.08\}$ (curve v). (b) Different vortex rings obtained with potential v: the number of vortices depends on local (random) variations of the vortex density (same conventions as in Fig. 2).
(BJR) equations when $\bar{\kappa} \approx 1 / \sqrt{2}^{6,7,9}$ while the first-order correction $\varphi_{1}=\left(P-R / 2 \bar{\kappa}^{2}\right)\left(1-\left|\varphi_{0}\right|^{2}\right) \varphi_{0}$ with

$$
P=p_{1}-p_{2} \quad \text { and } \quad R=r_{1}-r_{2} .
$$

At zeroth order, the free energy does not vary with the distance between the vortices since the energy of BJR solutions does not depend on the positions of the vortices. Its dependence on the separation is given at the first order of correction by the variation of $\int d^{2} x\left[\left(\bar{\kappa}^{2}-1 / 2+2 s\right)\left|\varphi_{0}\right|^{4}-s\left|\varphi_{0}\right|^{6}\right]$, which can be estimated in the limits of short and large separation as in Ref. 9. Here $s=\left(2 \bar{\kappa}^{2} P-R\right)^{2} / 4 q \bar{\kappa}^{2}$. We find ${ }^{26}$ that for large normalized Josephson coupling $q$ the vortex interaction is

$$
\begin{array}{cc}
\text { of type } 2 \text { when } & C \equiv \frac{q\left(1-1 / 2 \bar{\kappa}^{2}\right)}{2\left(P-R / 2 \bar{\kappa}^{2}\right)^{2}}>0.346, \\
\text { of type } 1.5 \text { when } & -0.4<C<0.346, \\
\text { of type } 1 \text { when } & C<-0.4 .
\end{array}
$$

$P$ and $R$ quantify the disparities between the two bands. When they are small or when $q$ is large, as in the vicinity of $T_{c}$, the ratio $C$ grows out of the bounds defining type 1.5 and superconductivity is either of type 1 or of type 2 . However, in the case $\bar{\kappa}=1 / \sqrt{2}, C$ is exactly zero, which means that the pair potential is always nonmonotonic despite a strong coupling.

Temperature dependence of the interaction. Since the average $\bar{\kappa}$ and the normalized Josephson coupling $q$ depend on temperature, the vortex-vortex interaction can also change with it. As an illustration, the temperature evolution of these quantities and of the ratio $C$ are plotted in Fig. 4(a) for different values of $\left\{\kappa_{1}, \kappa_{2}\right\}$, which yield type-1.5 superconductivity away from $T_{c}$. We use here parameters whose magnitudes are of the order of the experimental estimates for $\mathrm{MgB}_{2} .^{12,27,28}$ We adopted the model of temperature dependence for $\alpha_{n}$ from Refs. 27 and 17 with $\alpha_{1}$ becoming negative below $T_{1}=0.64 T_{c}$ and $\alpha_{2}$ becoming negative below $T_{2}=0.91 T_{c}$. At the critical temperature, $q$ diverges to infinity so the vortex-vortex interaction is either of type 1 or type 2 just
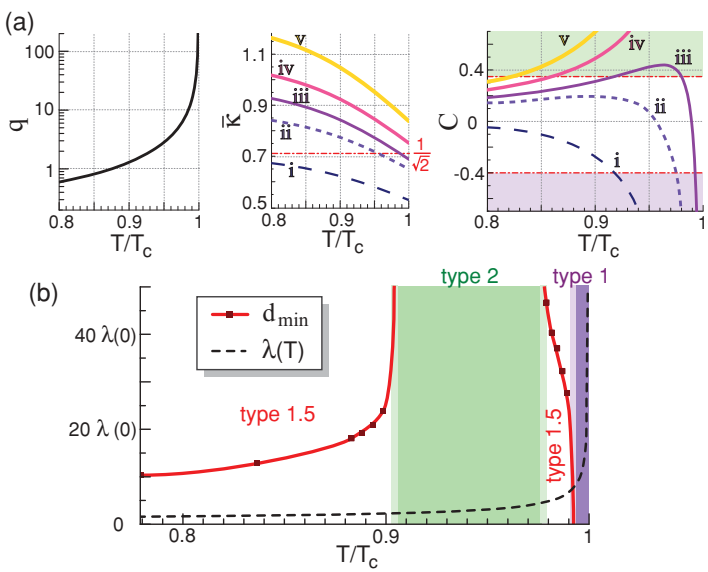

FIG. 4. (Color) (a) Temperature evolution of normalized Josephson coupling $q$, average GL parameter $\bar{\kappa}$, and vortexinteraction parameter $C$ for $\left\{\kappa_{1}, \kappa_{2}\right\}=$ (i) $\{0.5,1.5\}$, (ii) $\{0.6,2\}$, (iii) $\{0.6,2.65\}$, (iv) $\{0.65,3\}$, and (v) $\{0.7,4\}$. (b) Temperature evolution of the separation $d_{\min }$ at $U^{v v}$ minimum and of the magnetic penetration depth $\lambda$ for the parameter set (iii) in (a). 
below $T_{c}$. For the majority of parameter sets, there is only one change of behavior, type $1(2) \rightarrow$ type 1.5 , as temperature decreases. As shown by the example (iii) in Fig. 4(a), $\bar{\kappa}$ can pass by the value $1 / \sqrt{2}$ in the vicinity of $T_{c}$ while $q$ is large. In this case, $C$ can go out of the bounds (12) twice, which means an alternation of four regimes is possible. It was previously found $^{12,14}$ that a type-1.5 phase can survive above $\min \left(T_{1}, T_{2}\right)$, but a second interval above $\max \left(T_{1}, T_{2}\right)$ was unexpected. We numerically checked that for the set of parameters (iii) the phase diagram is composed by an unusual reentrant succession of four regimes: type $1 \rightarrow$ type $1.5 \rightarrow$ type $2 \rightarrow$ type 1.5 . The temperature dependence of the minimum position $d_{\min }$ and of the penetration depth $\lambda$ are plotted in Fig. 4(b). When the superconductor enters a type- 1 phase, the position of the potential minimum goes to zero while it diverges to infinity when entering a type-2 phase.
In conclusion, we find a condition on the material parameters for the appearance of type- 1.5 regime in the close vicinity of $T_{c}$ and we predict properties that clearly distinguish it from the vortex state of a conventional type- 2 superconductor. By numerical GL calculations of the vortex interaction potential and simulations of the resulting vortex dynamics, we find vortex-stabilized giant vortices, which survive in bunches and unusual rings of vortices in bulk multiband superconductors. The multicomponent character produces a complex phase diagram where type- 1.5 behavior can re-enter by alternating with type- 1 and type- 2 interludes in a succession of three regime transitions as temperature is changed.

Acknowledgments. The authors acknowledge financial support from the Methusalem Program by the Flemish Government.
*Corresponding author: victor.moshchalkov@ fys.kuleuven.be

${ }^{1}$ V. P. Mineev, Topologically Stable Defects and Solitons in Ordered Media (Harwood Academic Publishers, Amsterdam, 1998).

${ }^{2}$ C. O. Weiss and Y. Larionova, Rep. Prog. Phys. 70, 255 (2007).

${ }^{3}$ A. Ranjantie, Contemp. Phys. 44, 485 (2003); A.-C. Davis and T. W. B. Kibble, ibid. 46, 313 (2005); T. W. B. Kibble, Phys. Today 60, 47 (2007).

${ }^{4}$ B. Link, Astrophys. Space Sci. 308, 435 (2007); P. B. Jones, Mon. Not. R. Astron. Soc. 371, 1327 (2006).

${ }^{5}$ E. H. Brandt, Rep. Prog. Phys. 58, 1465 (1995).

${ }^{6}$ E. B. Bogomolnyi, Yad. Fiz. 24, 861 (1976) [Sov. J. Nucl. Phys. 24, 449 (1976)]; E. B. Bogomolnyi and A. I. Vainshtein, Yad. Fiz. 23, 1111 (1976) [Sov. J. Nucl. Phys. 23, 588 (1976)].

${ }^{7}$ L. Jacobs and C. Rebbi, Phys. Rev. B 19, 4486 (1979).

${ }^{8}$ U. Krägeloh, Phys. Status Solidi 42, 559 (1970).

${ }^{9}$ I. Luk'yanchuk, Phys. Rev. B 63, 174504 (2001).

${ }^{10}$ G. Blatter and V. Geshkenbein, Phys. Rev. Lett. 77, 4958 (1996).

${ }^{11}$ E. Babaev and M. Speight, Phys. Rev. B 72, 180502(R) (2005).

${ }^{12}$ V. V. Moshchalkov et al., Phys. Rev. Lett. 102, 117001 (2009); T. Nishio, V. H. Dao, Q. H. Chen, L. F. Chibotaru, K. Kadowaki, and V. V. Moshchalkov, Phys. Rev. B 81, 020506(R) (2010).

${ }^{13}$ J. Nagamatsu, N. Nakagawa, T. Muranaka, Y. Zenitani, and J. Akimitsu, Nature (London) 410, 63 (2001); A. Y. Liu, I. I. Mazin, and J. Kortus, Phys. Rev. Lett. 87, 087005 (2001); F. Bouquet, R. A. Fisher, N. E. Phillips, D. G. Hinks, and J. D. Jorgensen, ibid. 87, 047001 (2001); F. Giubileo et al., ibid. 87, 177008 (2001); P. Szabo et al., ibid. 87, 137005 (2001).

${ }^{14}$ E. Babaev, J. Carlstrom, and M. Speight, Phys. Rev. Lett. 105, 067003 (2010).

${ }^{15}$ Y. Kamihara, T. Watanabe, M. Hirano, and H. Hosono, J. Am. Chem. Soc. 130, 3296 (2008); F. Hunte, J. Jaroszynski, A. Gurevich, D. C. Larbalestier, R. Jin, A. S. Sefat, M. A. McGuire, B. C. Sales, D. K. Christen, and D. Mandrus, Nature (London) 453, 903 (2008); I. I. Mazin, D. J. Singh, M. D. Johannes, and M. H. Du, Phys. Rev. Lett. 101, 057003 (2008).

${ }^{16}$ F.-C. Hsu et al., Proc. Natl. Acad. Sci. USA. 105, 14262 (2008); R. Khasanov et al., Phys. Rev. Lett. 104, 087004 (2010); T. Hanaguri, S. Niitaka, K. Kuroki, and H. Takagi, Science 328, 474 (2010).
${ }^{17}$ M. E. Zhitomirsky and V. H. Dao, Phys. Rev. B 69, 054508 (2004); V. H. Dao and M. E. Zhitomirsky, Eur. Phys. J. B 44, 183 (2005).

${ }^{18}$ A. Gurevich, Physica C 456, 160 (2007).

${ }^{19}$ G. A. Vardanyan and D. M. Sedrakyan, Zh. Eksp. Teor. Fiz. 81, 1731 (1981) [Sov. Phys. JETP 54, 919 (1981)]; M. Alpar, S. Langer, and J. Sauls, Astrophys. J. 282, 533 (1984).

${ }^{20}$ The conjugate gradient method is stopped when the relative difference of energy between successive iterations is less than $5 \times 10^{-8}$. When compared to the exact value $F(d)=2$ for a single component at $\kappa=1 / \sqrt{2}[7]$, the relative error is $\approx 10^{-5}, 10^{-4}, 10^{-5}$, and $10^{-7}$ at $d / \lambda=1,2,5$, and 10 .

${ }^{21}$ The local ordering inside a vortex cluster is extremely fast in comparison to its migration because the intervortex force decreases exponentially with the separation. The stability of the final configuration was tested by running the simulation from this configuration for 200000 more time steps with step duration four times larger.

${ }^{22}$ A. Kanda, B. J. Baelus, F. M. Peeters, K. Kadowaki, and Y. Ootuka, Phys. Rev. Lett. 93, 257002 (2004); L. F. Chibotaru, V. H. Dao, and A. Ceulemans, Europhys. Lett. 78, 47001 (2007); L. F. Chibotaru and V. H. Dao, Phys. Rev. B 81, 020502(R) (2010).

${ }^{23}$ A. I. Buzdin, Phys. Rev. B 47, 11416 (1993); V. V. Moshchalkov et al., ibid. 54, 7385 (1996); R. B. G. Kramer, A. V. Silhanek, J. Van de Vondel, B. Raes, and V. V. Moshchalkov, Phys. Rev. Lett. 103, 067007 (2009).

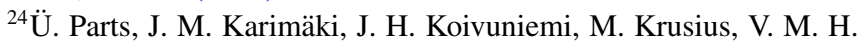
Ruutu, E. V. Thuneberg, and G. E. Volovik, Phys. Rev. Lett. 75, 3320 (1995); A. P. Finne, V. B. Eltsov, R. Hänninen, N. B. Kopnin, J. Kopu, M. Krusius, M. Tsubota, and G. E. Volovik, Rep. Prog. Phys. 69, 3157 (2006).

${ }^{25}$ G. E. Volovik and T. Sh. Misirpashaev, Pis'ma Zh. Eksp. Teor. Fiz. 51, 475 (1990) [JETP Lett. 51, 537 (1990)].

${ }^{26}$ The upper bound is equal to $\left(1-\delta \int \nabla \times a^{3} / \delta \int \nabla \times a^{2}\right) / 2$ that is numerically estimated to 0.346 ( $\delta \int$ stands for the variation of the integral as $d$ grows to infinity). The lower bound $3\left(\zeta_{8}^{(2)}-\right.$ $\left.\zeta_{6}^{(2)}\right) / 4\left(\zeta_{6}^{(2)}-\zeta_{4}^{(2)}\right)-1 \approx-0.4$ with the values of the constants $\zeta_{n}^{(2)}$ is given by Ref. 9 .

${ }^{27}$ M. Eisterer, M. Zehetmayer, H. W. Weber, and J. Karpinski, Phys. Rev. B 72, 134525 (2005); M. Eisterer, Supercond. Sci. Technol. 20, R47 (2007).

${ }^{28} \alpha_{1}(0) / \sqrt{\beta_{1}}=89.2, \quad \alpha_{2}(0) / \sqrt{\beta_{2}}=410.4, \quad$ and $\quad \gamma /\left(\beta_{1} \beta_{2}\right)^{1 / 4}=$ $38.9\left(\mathrm{~J}^{1 / 2} \mathrm{~m}^{-3 / 2}\right)$ 